
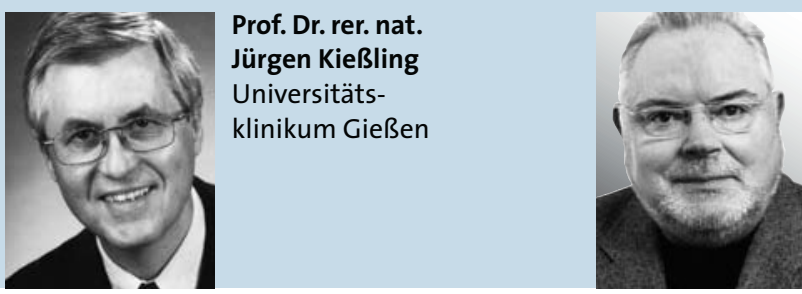

Dr. med.

Dieter Leithäuser

HNO-Arzt, Warburg

\section{Jodhaltige Kontrastmittel: Hypersensitivität trotz negativem Hauttest-Befund?}

Jodhaltige Röntgenkontrastmittel sind sichere Arzneimittel. Bei den weltweit über 75 Millionen Anwendungen pro Jahr ereignen sich dennoch vereinzelt leichte bis schwere Zwischenfälle aufgrund von Hypersensitivitätsreaktionen. In der Diagnostik der Hypersensitivitätsreaktionen haben sich Hauttests bewährt. Eine Studie untersuchte die klinische Relevanz negativer Befunde solcher Tests.

- ypersensitivitätsreaktionen gegen jodhaltige Kontrastmittel (iodinated contrast medium, ICM) lassen sich einteilen in Sofortreaktionen und NichtSofortreaktionen. Bei Sofortreaktionen treten die Symptome innerhalb der ersten Stunde nach Injektion auf, bei NichtSofortreaktionen innerhalb von sieben Tagen.

Patienten mit Verdacht auf ICM-Hypersensitivitätsreaktion werden meist mit Hauttests untersucht. Über die Zuverlässigkeit und klinische Relevanz der Hauttest-Ergebnisse ist bisher aber nicht viel bekannt. In einer retrospektiven Analyse wurde daher anhand von monozentrisch erhobenen Daten der negativ prädiktive Wert von ICM-Hauttests ermittelt. Dazu wurden 159 Patienten kontaktiert, die sich in den vergangenen neun Jahren aufgrund von Reaktionen nach ICM-Gabe einem ICM-Hauttest unterzogen hatten. In einem strukturierten Interview wurden sie nach möglichen Reaktionen bei einer Kontrastmittel-Reexposition gefragt.

75\% beantworteten die Fragen, $29 \mathrm{~Pa}$ tienten bejahten eine ICM-Reexposition.

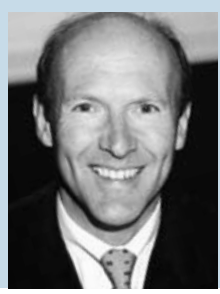

Prof. Dr. med. Ralph Mösges Universität Köln

Zwei Patienten hatten einen positiven Hauttest, erhielten bei einer erneuten Röntgenuntersuchung ein negativ getestetes Mittel und vertrugen dieses gut. Zwei negativ getestete Patienten berichteten über leichte Reaktionen bei einer Reexposition: Ein Patient hatte eine Sofortreaktion mit generalisierter Urtikaria über drei Tage, ein Patient eine leichte Nicht-Sofortreaktion mit Hautausschlag.

Fazit: In einer monozentrischen Untersuchung an Patienten mit Verdacht auf ICM-Hypersensitivität ergab sich für ICM-Hauttests ein negativ prädiktiver Wert von 96\% (95\%-Konfidenzintervall 89,9 bis 103,2). Zwei Patienten mit negativem Befund zeigten bei Reexposition leichte Reaktionen. Größere multizentrische Untersuchungen müssen diese Angaben nun bestätigen.

\section{bk}

Caimmi S et al. Clinical value of negative skin tests to iodinated contrast media. Clin Exp Allergy 2010; 40: 805--10 